\title{
Incidence of Inflammatory Bowel Disease in South Asian and Chinese People: A Population-Based Cohort Study from Ontario, Canada
}

\author{
Jasbir Dhaliwal (1D ${ }^{1-6}$ \\ Meltem Tuna' \\ Baiju R Shah ${ }^{1,7,8}$ \\ Sanjay Murthy ${ }^{1,9,10}$ \\ Emily Herrett ${ }^{4}$ \\ Anne M Griffiths ${ }^{2,3}$ \\ Eric I Benchimol (D) ${ }^{1-3,8}$ \\ 'ICES, Toronto, ON, Canada; ${ }^{2}$ SickKids \\ Inflammatory Bowel Disease Centre, \\ Division of Gastroenterology, Hepatology \\ and Nutrition, The Hospital for Sick \\ Children, Toronto, ON, Canada; \\ ${ }^{3}$ Department of Paediatrics, University of \\ Toronto, Toronto, ON, Canada; ${ }^{4}$ London \\ School of Hygiene and Tropical Medicine, \\ London, UK; ${ }^{5}$ Cincinnati Children's \\ Hospital Medical Center, Division of \\ Gastroenterology, Hepatology and \\ Nutrition, Cincinnati, OH, USA: \\ ${ }^{6}$ Department of Pediatrics, University of \\ Cincinnati, Cincinnati, OH, USA; \\ ${ }^{7}$ Sunnybrook Research Institute, \\ Sunnybrook Health Sciences Centre, \\ Toronto, ON, Canada; ${ }^{8}$ Institute of Health \\ Policy, Management and Evaluation, \\ University of Toronto, Toronto, ON, \\ Canada; ${ }^{9}$ The Ottawa Hospital IBD Centre, \\ The Ottawa Hospital and Ottawa Hospital \\ Research Institute, Ottawa, ON, Canada; \\ ${ }^{10}$ Department of Medicine and School of \\ Epidemiology and Public Health, University \\ of Ottawa, Ottawa, ON, Canada
}

Correspondence: Jasbir Dhaliwal Pediatrics, University of Cincinnati, Cincinnati Children's Hospital Medical Center, Division of Gastroenterology, Hepatology and Nutrition, Secondary Appointments in Division of Biomedical Informatics and James $M$ Anderson

Center for Health Systems Excellence,

3333 Burnet Avenue, Cincinnati, $\mathrm{OH}$, 45229, USA

Tel + I5I33789468

Email Jasbir.dhaliwal@cchmc.org
Background: Inflammatory bowel disease (IBD) is now a global disease with incidence increasing throughout Asia.

Aim: To determine the incidence of IBD among South Asians and Chinese people residing in Ontario, Canada's most populous province.

Methods: All incident cases of IBD in children (1994-2015) and adults (1999-2015) were identified from population-based health administrative data. We classified South Asian and Chinese ethnicity using immigration records and surnames. We determined standardized incidence of IBD and adjusted incidence rate ratio (aIRR) in South Asians and Chinese compared to the general population.

Results: Among 16,230,638 people living in Ontario, standardized incidence of IBD per 100,000 person-years was 24.7 (95\% CI 24.4-25.0), compared with 14.6 (95\% CI 13.7-15.5) in 982,472 South Asians and with 5.4 (95\% CI 4.8-5.9) in 764,397 Chinese. The risk of IBD in South Asians was comparable to the general population after adjusting for immigrant status and confounders (aIRR 1.03, 95\% CI 0.96-1.10). South Asians had a lower risk of Crohn's disease (CD) (aIRR 0.66, 95\% CI 0.60-0.77), but a higher risk of ulcerative colitis (UC) (aIRR $1.47,95 \%$ CI 1.34-1.61). Chinese people had much lower rates of IBD (aIRR 0.24, 95\% CI $0.20-0.28$ ), CD (aIRR 0.21, 95\% CI 0.17-0.26), and UC (aIRR 0.28, 95\% CI 0.23-0.25).

Conclusion: Canadians of South Asian ethnicity had a similarly high risk of developing IBD compared to other Canadians, and a higher risk of developing UC, a finding distinct from the Chinese population. Our findings indicate the importance of genetic and environmental risk factors in people of Asian origin who live in the Western world.

Keywords: inflammatory bowel disease, ulcerative colitis, Crohn's disease, incidence, ethnicity, epidemiology, South Asian, Chinese

\section{Introduction}

Inflammatory bowel disease (IBD) is characterized by chronic inflammation of the bowel and comprised of two major sub-types, Crohn's disease (CD) and ulcerative colitis (UC). ${ }^{1}$ Whereas incidence of IBD is stabilizing in the Western World, it is rapidly increasing in newly industrialized countries. ${ }^{2}$ Canada has among the highest rates in the world, and prevalence is projected to rise from $0.7 \%$ in 2018 to $1 \%$ in $2030{ }^{3}$ Ontario, Canada's most populous province, has a highly diverse population, with visible minorities representing $29 \%$ of residents. ${ }^{4,5}$ Previous population-based studies from Ontario have demonstrated lower rates of IBD among immigrants from South and East Asia relative to non-immigrants, but rates among Canadian-born children of South 
Asian immigrants were comparable to the children of nonimmigrants. ${ }^{6}$ However, this study evaluated only immigrants, and not the influence of ethnicity in people who were not immigrants. To understand the role of environmental risk factors in the pathophysiology of IBD, it is important to understand the role of ethnicity in IBD epidemiology. In this population-based study, we described differences in the incidence of IBD in people of South Asian and Chinese ethnicity compared to other residents of Ontario.

\section{Methods}

\section{Study Design, Setting and Study Population}

We conducted a cohort study using the population-based health administrative data for Ontario, Canada (population 14.4 million). We included all residents of Ontario registered for universal healthcare ( $>99 \%$ of the population) from fiscal year (FY) 1991 to 2018 (ie, April 1, 1991 to March 31, 2019). We excluded individuals with missing date of birth or missing valid ICES unique encrypted identification number (IKN), or those who left Ontario and then returned during the study period (due to incomplete administrative data). We excluded those with missing data for both exposure (ethnicity) and independent variables (confounders). Ontario residents were followed forward within the databases to determine whether they were diagnosed with IBD before
April 1, 2019. This study was approved by the research ethics boards of the London School of Hygiene and Tropical Medicine (21620/RR/18073) and the Children's Hospital of Eastern Ontario (19/08PE).

\section{Data Sources}

Ontario health administrative data is held at ICES, an independent, non-profit research institute funded by an annual grant from the Ontario Ministry of Health $(\mathrm{MOH})$ and the Ministry of Long-Term Care (MLTC). The datasets were linked deterministically using unique encoded identifiers (IKN) and analyzed at ICES. We identified all individuals from the Registered Persons Database (RPDB), which contains information on all legal residents of Ontario registered for universal health care under the Ontario Health Insurance Plan (OHIP). RPBD also contains information including date of birth, location of residence (postal codes), sex and mean neighbourhood income quintile.

We linked three databases to characterize the South Asian and Chinese populations, and other Ontario residents defined as "general" (Figure 1). The ETHNIC database assigns ethnicity based on surnames. ${ }^{7}$ South Asian surnames are largely of Hindu, Sikh, Bhutan and Sri Lankan origin, and surnames of Christian and Muslim origin were excluded because of commonality with other ethnic groups. The ETHNIC database has a positive predictive value (PPV) of $89.3 \%$ and

\section{Study population}

\section{Ethnicity assignment}

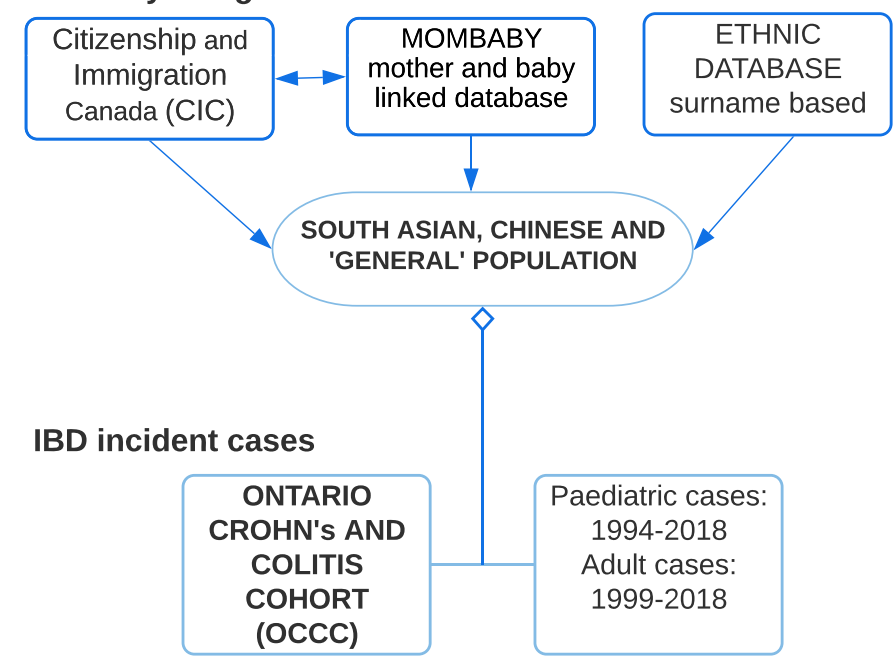

\section{Covariates}

1. Immigration status $(\mathrm{ClC})$ 2. Mean neighborhood income quintile on date of diagnosis (from RPDB) 3.Rurality index of Ontario (RIO)* score on date of diagnosis (from RPDB)

Age $(<18 y, 18-65,>65)$ and Sex Registered Persons Database (RPBD) 
sensitivity of $50.4 \%$ to identify South Asian individuals, and PPV of $91.9 \%$ and sensitivity of $80.2 \%$ to identify people of Chinese origin. In addition, to address possible misclassification by the ETHNIC database, we linked immigration data from Immigration, Refugees and Citizenship Canada (IRCC)'s Permanent Residents Database ${ }^{8}$ and defined ethnicity based on region of birth. The Ontario portion of IRCC data includes all immigrants arriving to Ontario after January 1, 1985 (people who immigrated prior to 1985 are misclassified as non-immigrants). As per World Bank region classification, ${ }^{9}$ individuals were labelled as South Asian if they were born in India, Pakistan, Nepal, Bangladesh, Bhutan or Sri Lanka. Individuals were labelled as Chinese if born in China, Hong Kong or Taiwan. The Canadian-born children of immigrant mothers were identified by linking female immigrants to the MOMBABY database. MOMBABY links maternal and new-born records of hospital births from 1988 onward. ${ }^{10,11}$

We used the Ontario Crohn's and Colitis Cohort (OCCC) to identify all incident cases of IBD, and to distinguish $\mathrm{CD}$ from $\mathrm{UC} .^{12,13} \mathrm{OCCC}$ is a complete cohort of IBD patients living in Ontario from 1991 onwards. Validated lookback periods without health-care contacts for IBD were required to distinguish incident from prevalent cases (except for people born after April 1, 1991); 3 years for paediatric-onset IBD and 8 years for adult- or elderly-onset IBD. Therefore, the paediatric incident cohort started April 1, 1994; the adult/elderly incident cases starting April 1, 1999. The algorithm to identify children with IBD (aged $6 \mathrm{mo}$ to $<18$ years) was found to have a sensitivity of $89.6-91.1 \%$, specificity $>99.9 \%$, PPV of $59.2-76.0 \%$ and negative predictive value (NPV) $>99.9 \%{ }^{12}$ In adults (aged 18 to $<65$ years) a sensitivity of $76.8-92.3 \%$, specificity of $96.2-99.1 \%$, PPV of $81.4 \%$ and NPV of $95.0 \% .{ }^{13}$ The algorithm in seniors ( $>65$ years) had a sensitivity of $52.2-53.7 \%$, specificity of 98.8 $99.3 \%$, PPV of $76.3 \%$, and NPV of $98.0 \% .{ }^{13}$ IBD incident patients were further stratified as $\mathrm{CD}$, UC or IBD type unclassifiable using a validated algorithm. ${ }^{12,13}$

\section{Exposure Ascertainment}

We identified two ethnic groups using the abovementioned databases, South Asian and Chinese, and the remaining population defined as the "general" cohort served as the comparative reference population. If ethnicity determined by immigration data differed from the ETHNIC database, ethnicity was assigned using country of birth from immigration data (Figure 1). We assessed the degree of concordance in assigning ethnicity between the ETHNIC database and IRCC immigration data. The IRCC and ETHNIC databases were highly concordant among Chinese individuals (93.5\% concordance), but less concordant among South Asians $(37.7 \%$ concordance) (Supplementary Table 1).

\section{Outcome Ascertainment}

The outcome of interest was the incident diagnosis of IBD, identified using the OCCC with the validated algorithms described above.

\section{Independent Variables (Confounders)}

Multivariable models adjusted for the following a priori determined independent variables, identified as possible confounders using clinical knowledge and previous research: immigration status, ${ }^{12}$ sex, neighbourhood income quintile at cohort entry ${ }^{14}$ and Rurality Index of Ontario (RIO) at the time of cohort entry. ${ }^{15}$ RIO is an ordinal variable measures the degree of rurality and consists of three broad components, measure of community population and density, measure of travel to nearest basic referral centre, and measure of travel time to nearest advanced centre. ${ }^{16}$ The RIO is a normalised scale ranging from 0 to 100 , with a score of 0 being the least rural.

\section{Statistical Analysis}

Standardized incidence was calculated per 100,000 person years (PYs) of follow-up, with 95\% confidence intervals (CI) based on gamma distribution, using age-appropriate standard populations. We used the Canadian Census data from 2016 to determine the annual intercensal population estimates. ${ }^{17}$ To assess risk of IBD (including CD and UC subtypes), we compared incidence in ethnic groups. We calculated standardized incidence rate ratio (IRR) adjusted for age and sex with 95\% CI using Poisson regression. We also determined adjusted IRR with 95\% CI using negative binomial regression model, controlling for sex (female reference), immigration category (non-immigrant reference), income quintile (lowest income quintile as reference) and RIO rurality index at the time of cohort entry.

To determine the possible protective association of immigration status on the risk of IBD, we used multivariable models without controlling for immigration status (model 1) and controlling for immigration status (model 2). Age was categorized into three levels: i) 6 months to $<18$ years (paediatric); ii) 18 years to $<65$ years (adult); iii) $\geq 65$ years (senior). Interaction between 
age and the main exposure ethnicity (age*ethnic) was included as an independent variable in model 3. If the interaction term was significant $(\mathrm{p}<0.05)$, the analysis was stratified by age group (model 4 ). We described normally distributed continuous variables as means and standard deviations (SD), and categorical variables were expressed as frequency and proportions. Statistical analyses were performed using Stata version 15 (StataCorp) and using SAS version 9.4 (SAS Institute, NC).

\section{Results}

\section{Study Participants}

Of the 16,230,638 residents of Ontario included in our cohort, 982,472 (6.1\%) were South Asian and 764,397 (4.7\%) Chinese. Over the follow-up period, 1644 South Asians and 444 Chinese developed IBD, and this accounted for $3.4 \%$ and $0.9 \%$ of total incident cases. See Table 1 for the descriptive characteristics of the IBD cohort. Males predominated among South Asian and Chinese individuals with IBD comprising, respectively, $56 \%$ and $58 \%$ of these cohorts. The IBD South Asian cohort was younger relative to the general and Chinese IBD cohorts. Non-immigrant South Asian people were diagnosed with IBD at 30.3 $\pm 18.7 \mathrm{y}$, compared to $39.1 \pm 18.7 \mathrm{y}$ in South Asian immigrants. There was no difference observed among immigrants and non-immigrants in the Chinese and general IBD cohorts (Supplementary Table 2). Less than 5\% of the IBD patients in the general cohort were immigrants, compared to approximately $40 \%$ of South Asians, and $30 \%$ of Chinese IBD patients. No person was missing age or sex, and immigration status. Two percent of records were missing mean neighborhood income quintile and $0.5 \%$ were missing RIO score. These residents were removed from the analyses.

\section{Standardized Incidence Rates}

Standardized incidence of IBD per 100,000 PYs in the general cohort was 24.7 (95\% CI 24.4-25.0), compared with 14.6 (95\% CI 13.7-15.5) in South Asians and with 5.4 (95\% CI 4.8-5.9) in Chinese. The standardized incidence of CD per 100,000 PYs among the general cohort $(10.6,95 \%$ CI 10.4-10.7) was significantly higher than in the South Asians and Chinese populations (South Asians: 4.28, 95\% CI 3.804.76; Chinese: $1.58,95 \%$ CI 1.26-1.90). The standardized

Table I Descriptive Characteristics of the IBD Population

\begin{tabular}{|c|c|c|c|}
\hline & General Population & South Asian & Chinese \\
\hline IBD incident cases & 45,508 & 1644 & 444 \\
\hline Follow-up person-years & $10.0 \pm 5.3$ & $10.6 \pm 4.88$ & $10.5 \pm 5.04$ \\
\hline Male & $21,959(48.3 \%)$ & $912(55.5 \%)$ & $259(58.3 \%)$ \\
\hline Age at diagnosis $(y)$ & $39.5 \pm 19.3$ & $33.7 \pm 18.0$ & $41.1 \pm 18.2$ \\
\hline Age at diagnosis of immigrant (y) & $37.5 \pm 15.6$ & $39.1 \pm 15.3$ & $41.3 \pm 14.7$ \\
\hline Age at diagnosis of non-immigrant $(y)$ & $39.6 \pm 19.4$ & $30.3 \pm 18.7$ & $41.0 \pm 19.5$ \\
\hline \multicolumn{4}{|l|}{ Age at diagnosis $(y)$} \\
\hline$<18 y$ & $6305(13.9 \%)$ & $377(22.9 \%)$ & $43(9.7 \%)$ \\
\hline $18-<65 y$ & 33,831 (74.3\%) & II 73 (7I.4\%) & $356(80.2 \%)$ \\
\hline$\geq 65 y$ & 5372 (II.8\%) & 94 (5.7\%) & $45(10.1 \%)$ \\
\hline Rural & $7525(16.6 \%)$ & 64 (3.9\%) & 14 (3.2\%) \\
\hline \multicolumn{4}{|l|}{ Income quintile } \\
\hline I & $8526(19.2 \%)$ & $519(31.9 \%)$ & 98 (22.5\%) \\
\hline 2 & 8839 (19.9\%) & $415(25.5 \%)$ & $95(21.8 \%)$ \\
\hline 3 & $8954(20.1 \%)$ & $320(19.7 \%)$ & $97(22.2 \%)$ \\
\hline 4 & 9183 (20.7\%) & $240(14.8 \%)$ & $87(20.0 \%)$ \\
\hline 5 & 8947 (20.1\%) & $|3|(8.1 \%)$ & 59 (I3.5\%) \\
\hline Immigrant & $1860(4.1 \%)$ & $642(39.1 \%)$ & I 32 (29.7\%) \\
\hline Rurality Index 2008 & $11.2 \pm 17.9$ & $1.7 \pm 5.0$ & $2.4 \pm 8.2$ \\
\hline
\end{tabular}

Notes: Values are presented as $\mathrm{N}(\%)$, mean \pm standard deviation. 
incidence of UC per 100,000 PYs among South Asians (9.56, 95\% CI 8.83-10.3) and Chinese (3.54, 95\% CI 3.06-4.02) was twice the rate of $\mathrm{CD}$. The incidence rates and rate ratios by IBD type are reported in Tables 2 and 3 .

\section{Incidence in South Asians Compared with the General Population}

The risk of IBD in South Asians was lower compared to the general population prior to adjusting for immigration status (model 1; adjusted IRR (aIRR)0.73, 95\% CI 0.69-0.76), but the same as the general population after adjusting for immigration in the model (model 2; aIRR 1.00, 95\% CI 0.95-1.05) (Table 4). After adjustment for immigration status, only South Asian adults had a higher risk of IBD (aIRR 1.10, 95\% CI
1.02-1.18), not children (0.98 95\% CI $0.91-1.05)$ or seniors (0.38 95\% CI 0.14-1.06) relative to the general population. Furthermore, immigration was significantly associated with a lower risk of both CD and UC (Table 4 and Supplementary Table 3). The incidence of CD in South Asians was lower compared to the general population, irrespective of age (aIRR $0.66,95 \%$ CI $0.60-0.76$ ), whilst the risk of UC was higher in South Asian especially among children and adult South Asians (aIRR 1.47, 95\% CI 1.34-1.61; children aIRR 1.36, 95\% CI 1.24-1.49; adults aIRR 1.30, 95\% CI 1.19-1.43). South Asians were more likely to develop UC than CD (aIRR 1.51, 95\% CI 1.38-1.66) (Table 5 and Supplementary Table 6). We observed a difference in IRR by age, and the incidence rate decreased with increasing age among South Asians relative to both the

Table 2 Standardized Incidence Rate per 100,000 Person-Years (PYs) by Ethnicity (1999-20I5) and IBD Type

\begin{tabular}{|c|c|c|c|c|c|}
\hline & $\begin{array}{l}\text { Number of } \\
\text { Incident } \\
\text { Cases }\end{array}$ & Total Population & PYs & $\begin{array}{c}\text { Crude Incidence } \\
\text { Rate per } 100,000 \\
\text { PYs }(95 \% \mathrm{CI})\end{array}$ & $\begin{array}{c}\text { Standardized } \\
\text { Incidence Rate per } \\
100,000 \text { (PYs) }(95 \% \\
\text { Cl) }\end{array}$ \\
\hline \multicolumn{6}{|c|}{ Inflammatory bowel disease } \\
\hline General population & 44,325 & $14,483,769$ & $178,56 \mid, 105$ & $24.8(24.6-25.1)$ & $24.7(24.4-25.0)$ \\
\hline South Asian & 1610 & 982,472 & $9,719,040$ & $16.6(15.8-17.4)$ & $14.6(\mid 3.7-15.5)$ \\
\hline Chinese & 439 & 764,397 & $8,393,123$ & $5.2(5.72-4.74)$ & $5.4(4.8-5.9)$ \\
\hline \multicolumn{6}{|l|}{ Crohn's Disease } \\
\hline General population & 20,425 & $|5,466,24|$ & $178,357,430$ & $11.5(11.3-11.6)$ & $10.6(10.4-10.7)$ \\
\hline South Asian & 493 & 982,472 & $9,708,882$ & $5.08(4.65-5.55)$ & $4.28(3.80-4.76)$ \\
\hline Chinese & 138 & 764,397 & $8,390,392$ & $1.64(1.37-1.92)$ & $1.58(1.26-1.90)$ \\
\hline \multicolumn{6}{|l|}{ Ulcerative Colitis } \\
\hline General population & 21,635 & $|5,466,24|$ & $178,372,216$ & $12.1(12.0-12.3)$ & $12.9(12.7-13.1)$ \\
\hline South Asian & 1027 & 982,472 & $9,713,445$ & $10.6(9.95-11.2)$ & $9.56(8.83-10.3)$ \\
\hline Chinese & 283 & 764,397 & $8,391,614$ & $3.37(3.00-3.79)$ & $3.54(3.06-4.02)$ \\
\hline
\end{tabular}

Table 3 Standardized Incidence Rate Ratio by Ethnicity and IBD Type

\begin{tabular}{|l|c|c|c|c|c|c|}
\hline \multirow{2}{*}{} & \multicolumn{2}{|c|}{ Inflammatory Bowel Disease } & \multicolumn{2}{|c|}{ Crohn's Disease } & \multicolumn{2}{c|}{ Ulcerative Colitis } \\
\cline { 2 - 7 } & $\begin{array}{c}\text { Crude IRR } \\
\mathbf{( 9 5 \% ~ C l )}\end{array}$ & $\begin{array}{c}\text { Standardized IRR } \\
\mathbf{( 9 5 \% ~ C l )}\end{array}$ & $\begin{array}{c}\text { Crude IRR } \\
\mathbf{( 9 5 \% ~ C l )}\end{array}$ & $\begin{array}{c}\text { Standardized IRR } \\
\mathbf{( 9 5 \% ~ C l )}\end{array}$ & $\begin{array}{c}\text { Crude IRR } \\
\mathbf{( 9 5 \% ~ C l )}\end{array}$ & $\begin{array}{c}\text { Standardized IRR } \\
\mathbf{( 9 5 \% ~ C l )}\end{array}$ \\
\hline $\begin{array}{l}\text { South Asian vs } \\
\text { general }\end{array}$ & $0.69(0.66-0.73)$ & $0.59(0.55-0.63)$ & $0.46(0.42-0.49)$ & $0.41(0.36-0.45)$ & $0.91(0.86-0.97)$ & $0.74(0.69-0.80)$ \\
\hline $\begin{array}{l}\text { Chinese vs } \\
\text { general }\end{array}$ & $0.21(0.19-0.24)$ & $0.22(0.19-0.24)$ & $0.15(0.12-0.17)$ & $0.15(0.12-0.18)$ & $0.28(0.25-0.32)$ & $0.28(0.24-0.32)$ \\
\hline $\begin{array}{l}\text { South Asian vs } \\
\text { Chinese }\end{array}$ & $3.24(2.92-3.60)$ & $2.72(2.40-3.09)$ & $3.10(2.58-3.74)$ & $2.71(2.15-3.41)$ & $3.24(2.84-3.69)$ & $2.70(2.31-3.15)$ \\
\hline
\end{tabular}

Notes: General the comparative reference population cohort.

Abbreviation: IRR, incidence rate ratio. 
Table 4 Incidence Rate Ratio Models of Inflammatory Bowel Disease, Crohn's Disease and Ulcerative Colitis, Comparing: South Asian Compared with General Population, Chinese with General and South Asian with Chinese

\begin{tabular}{|c|c|c|c|c|c|c|}
\hline & \multicolumn{2}{|c|}{ Inflammatory Bowel Disease } & \multicolumn{2}{|c|}{ Crohn's Disease } & \multicolumn{2}{|c|}{ Ulcerative Colitis } \\
\hline & IRR (95\% CI) & SE & IRR (95\% CI) & SE & IRR (95\% CI) & SE \\
\hline \multicolumn{7}{|c|}{ South Asian (Ref: General) } \\
\hline Model I (-immigration status) ${ }^{\dagger}$ & $0.73(0.69-0.76)$ & 0.02 & $0.48(0.44-0.52)$ & 0.02 & $0.95(0.89-1.01)$ & 0.03 \\
\hline Model 2 (+ immigration status) & $1.0(0.95-1.05)$ & 0.03 & $0.70(0.64-0.77)$ & 0.03 & $1.25(1.17-1.33)$ & 0.04 \\
\hline Model 3 (*agegp) & $1.03(0.96-1.10)$ & 0.04 & $0.66(0.60-0.76)$ & 0.04 & $1.47(1.34-1.61)$ & 0.07 \\
\hline Model 4a $(<18 y)$ & $0.98(0.91-1.05)$ & 0.04 & $0.66(0.58-0.74)$ & 0.04 & $1.36(1.24-1.49)$ & 0.07 \\
\hline Model 4b $(18-<65 y)$ & $1.10(1.02-1.18)$ & 0.04 & $0.77(0.67-0.89)$ & 0.06 & $1.30(1.19-1.43)$ & 0.06 \\
\hline Model 4c (>65y) & $0.38(0.14-1.06)$ & 0.20 & $0.19(0.02-1.64)$ & 0.21 & $0.61(0.18-2.02)$ & 0.37 \\
\hline \multicolumn{7}{|c|}{ Chinese (Ref: General) } \\
\hline Model I (-immigration status) $\dagger$ & $0.22(0.20-0.24)$ & 0.01 & $0.15(0.13-1.18)$ & 0.01 & $0.28(0.25-0.32)$ & 0.02 \\
\hline Model 2 (+immigration status) & $0.26(0.24-0.29)$ & 0.01 & $0.19(0.16-0.23)$ & 0.02 & $0.33(0.29-0.37)$ & 0.02 \\
\hline Model 3 (*agegp) & $0.24(0.20-0.28)$ & 0.02 & $0.21(0.17-0.26)$ & 0.02 & $0.28(0.23-0.35)$ & 0.03 \\
\hline Model 4a $(<18 y)$ & $0.23(0.20-0.27)$ & 0.02 & $0.20(0.16-0.26)$ & 0.02 & $0.27(0.2 \mathrm{I}-0.33)$ & 0.03 \\
\hline Model 4b $(18-<65 y)$ & $0.30(0.26-0.33)$ & 0.02 & $0.18(0.14-0.23)$ & 0.02 & $0.38(0.33-0.44)$ & 0.03 \\
\hline Model 4c (>65y) & $0.22(0.09-0.52)$ & 0.10 & $0.19(0.04-0.88)$ & 0.15 & $0.27(0.09-0.8 \mathrm{I})$ & 0.15 \\
\hline \multicolumn{7}{|c|}{ South Asian (Ref: Chinese) } \\
\hline Model I(-immigration status) $\dagger$ & $3.37(3.03-3.75)$ & 0.18 & $3.15(2.6 I-3.8 I)$ & 0.30 & $3.40(2.98-3.89)$ & 0.23 \\
\hline Model 2 (+immigration status) & $3.73(3.35-4.15)$ & 0.20 & $3.52(2.90-4.24)$ & 0.34 & $3.76(3.29-4.30)$ & 0.26 \\
\hline Model 3 (*agegp) & $4.29(3.62-5.08)$ & 0.37 & $3.26(2.45-4.09)$ & 0.41 & $5.19(4.01-6.57)$ & 0.63 \\
\hline Model 4a $(<18 y)$ & $4.25(3.58-5.04)$ & 0.37 & $3.19(2.46-4.13)$ & 0.42 & $5.13(4.04-6.51)$ & 0.62 \\
\hline Model 4b $(18-<65 y)$ & $3.54(3.07-4.08)$ & 0.26 & $3.76(2.82-5.02)$ & 0.55 & $3.38(2.85-4.01)$ & 0.29 \\
\hline Model 4c (>65y) & $2.24(0.61-8.19)$ & 1.48 & $1.35(0.12-15.0)$ & 1.66 & $3.63(0.5 \mathrm{I}-25.9)$ & 3.64 \\
\hline
\end{tabular}

Notes: All models adjusted for covariates: immigration status texclusion of immigration status from model I), male (female as reference), neighbourhood income quintile (lowest income quintile as reference) and RIO2008, *Agegp interaction term.

Abbreviations: $\mathrm{Cl}$, confidence intervals; IRR, incidence rate; SE, standard error.

Table 5 Incidence Rate Ratio Models of Ulcerative Colitis Relative to Crohn's Disease

\begin{tabular}{|l|c|c|c|c|c|c|}
\hline \multirow{2}{*}{} & \multicolumn{2}{|c|}{ South Asian General (REF) } & \multicolumn{2}{c|}{ Chinese General (REF) } & \multicolumn{2}{c|}{ South Asian Chinese (REF) } \\
\cline { 2 - 7 } & IRR (95\% CI) & SE & IRR (95\% CI) & SE & IRR (95\% CI) & SE \\
\hline Model I (-immigration status) & $1.25(1.17-1.33)$ & 0.04 & $1.24(1.09-1.39)$ & 0.07 & $1.00(0.88-1.15)$ & 0.07 \\
Model 2 (+immigration status) & $1.19(1.12-1.28)$ & 0.04 & $1.20(1.06-1.35)$ & 0.07 & $0.99(0.87-1.14)$ & 0.07 \\
Model 3 (*agegp) & 1.51 (I.38-1.66) & 0.07 & $1.09(0.87-1.36)$ & 0.12 & $1.36(1.08-1.73)$ & 0.16 \\
\hline
\end{tabular}

Notes: All models adjusted for covariates: immigration status ('exclusion of immigration status from model I), male (female as reference), neighbourhood income quintile (lowest income quintile as reference) and RIO2008. *Agegp interaction term.

Abbreviations: $\mathrm{Cl}$, confidence intervals; IRR, incidence rate; SE, standard error; REF, reference group.

general and Chinese population (Figure 2 and Supplementary Figures 1, 2, 5, and 6).

\section{Incidence in Chinese population Compared with the General Population}

The risk of IBD was much lower in Chinese population, irrespective of age, compared to the general population (aIRR 0.24, 95\% CI 0.20-0.28). This lower risk was similar in UC (aIRR $0.28,95 \%$ CI $0.23-0.35$ ) and CD (aIRR $\quad 0.21 \quad 95 \% \quad$ CI $\quad 0.17-0.26$ ) (Table 4 and Supplementary Table 4). Immigration status did not significantly change the aIRR in the models (comparing model 1 with model 2). The IRR stratified by age and sex are demonstrated in Figure 2 (Supplementary Figures 3 and 4 ), with no noticeable difference in IRR by age. 


\section{Incidence in South Asian Population Compared with the Chinese Population}

The risk of IBD among South Asian children and adults was approximately four times higher than the Chinese population, irrespective of IBD type (Table 4 and Supplementary Table 5). South Asians had a greater risk of ulcerative colitis (vs CD) compared to Chinese population (IRR1.36, 95\% CI 1.08-1.73) (Table 5), and the risk of IBD (CD/UC) among South Asian relative to Chinese decreased with age (Figure 2 and Supplementary Figures 5 and 6).

\section{Discussion}

In this population-based study, the risk of developing IBD among South Asian people was comparable to the general population after adjusting for immigration status. This was not the case for Chinese residents, who had a significantly lower risk of IBD in both immigrants and non-immigrants. Notably, after adjustment for immigration status and other confounders, the risk of UC in South Asians was 1.5-fold higher than the general population. There was a male predominance in both the Chinese and South Asian IBD cohorts compared to the general population cohort. We also observed a distinct age-risk relationship in South Asians only, with higher relative incidence in younger people. Furthermore, South Asian and Chinese people were more likely to develop $\mathrm{UC}$ relative to $\mathrm{CD}$ compared to the general population. Our study supports the hypothesis that the likelihood of IBD, specifically UC, among the South Asian population is greater than in the Chinese population, and like the high incidence rates of IBD described in the general Canadian population.

The landscape of IBD is evolving; in the Western world, incidence is stabilizing and regions transitioning into a compounding prevalence stage, whilst newly industrialised countries entering acceleration in incidence stage, associated with rising incidence and low prevalence. ${ }^{2,18}$ This is particularly evident in South Asia, with countries such as India approaching the incidence of IBD comparable to the West. ${ }^{2}$ The present study further supports the notion that the risk of IBD increases in certain migrant groups from countries with lower IBD prevalence once they arrive to higher prevalence countries such as Canada, USA and the United Kingdom. ${ }^{6,19-21}$ The predominance of UC observed in both South Asian and Chinese IBD populations is consistent with other population-based studies from the Asia-Pacific region. ${ }^{22}$ The comparatively lower $\mathrm{CD}$ incidence rates may be attributed to the lack of a genotypic association. CDassociated polymorphisms in the NOD2/CARD15 gene are strongly associated with ileal $\mathrm{CD},{ }^{23}$ but the higher prevalence of these common risk alleles of European origin has not been widely observed in South Asians ${ }^{24}$ and Chinese patients. ${ }^{25}$ Likewise, population studies in the United Kingdom ${ }^{19,20}$ have observed a higher risk of UC among adult South Asians. In addition, using self-reported ethnicity in a prospective Canadian pediatric inception cohort study, we recently observed a predominance of UC in South Asian children and adolescents with IBD. ${ }^{26}$ We previously demonstrated that earlier age of immigration was associated with an increased risk of IBD, with risk increasing by $14 \%$ for every younger decade of life at immigration. ${ }^{6}$ Similarly, in our cohort, South Asians born in Canada with IBD presented at an earlier age relative to South Asian immigrants and the Chinese and general populations. These findings support that earlier exposure to the Canadian environment increases the risk of IBD and moreover UC, in this population.
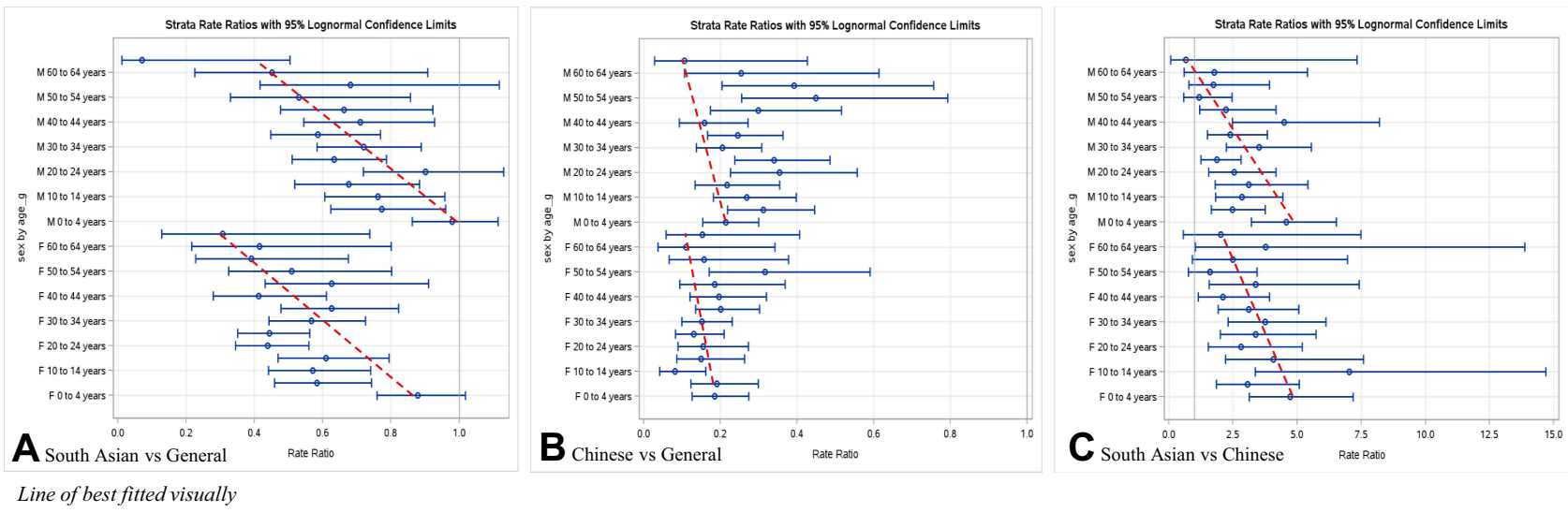

Figure 2 Inflammatory bowel disease unadjusted incidence rate ratio stratified by age and sex; [A] South Asian compared with general population; [B] Chinese compared with "general" population; [C] South Asian compared with Chinese population. 
It has been speculated that early exposure to environmental risk factors modifies the risk of developing IBD in genetically susceptible individuals. ${ }^{27,28}$ Notably, the emergence of IBD in Asian and Asian-Pacific countries associated with Westernisation signifies the important role of environmental factors. ${ }^{2,22}$ A recent meta-analysis of observational studies identified nine risk (smoking, urban living, vitamin D deficiency) and seven protective environmental factors (breastfeeding, tea, physical activity), with moderate to high epidemiologic evidence of association. ${ }^{27}$ However, evidence of association does not equate to causality and factors such as serum vitamin levels and microorganisms may be a consequence of reverse causation. The degree to which, and pathophysiologic mechanism by which, these environmental exposures contribute to risk of developing IBD remains to be elucidated. The relative contribution of environmental risk factors may vary according to the population studied. In our study, the risk of IBD among the Chinese population remained low even after adjusting for immigration, suggesting that exposure to the Canadian environment, even in people born in Canada, may not trigger IBD in people of Chinese origin. Protective genetic or microbiome factors may mitigate such environmental effects. These findings highlight the heterogeneity of the disease and the complex interplay of environmental, microbial, immunological and genetic factors, and future research should further assess the interplay of these factors among various ethno-cultural groups.

The strengths of this study include its large sample size and population-based study design. Linkage of multiple health administrative databases allowed us to calculate the incidence of IBD within ethnic groups in Ontario, and to adjust for known confounders. The use of health administrative data presents some limitations, including risk of misclassification bias, which could result from miscategorising people as having or not having IBD. However, we used validated algorithms in our study to identify patients with IBD, differentiate incident from prevalent cases, and classify IBD patients as having CD or UC. ${ }^{12,13}$ The IBD algorithms were not specifically validated in immigrants and we may have differentially misclassified people in these groups as having or not having IBD based on their health services utilisation patterns. However, we previously reported that health services utilisation was not different among immigrants with IBD compared to nonimmigrants, reducing the risk of differential misclassification. $^{29}$
Another limitation of this study is the assignment of South Asian ethnicity based on a surname-based algorithm, which was an excellent PPV but a sensitivity of $50.4 \%{ }^{7}$ The lower sensitivity was likely due to the exclusion of South Asian people with Muslim or European surnames, as to avoid misclassification. To overcome this weakness, we linked immigration and birth data to assign ethnicity. Immigrants from South Asia or China/ Hong Kong were classified as being of South Asian and Chinese ethnicity, respectively, even when the surname algorithm did not classify them as such. The children of immigrant mothers from those regions were classified with the same approach. However, immigration data were only available from 1985, and include only immigrants whose first place of landing was Ontario. Therefore, there is still the risk of misclassification. In our study, South Asians comprised $6.1 \%$ and Chinese $4.7 \%$ of the entire Ontario population, which was analogous to the 2006 and 2016 Canadian census (South Asian 6.6\% and 8.7\%, Chinese $4.7 \%$ and $5.7 \%$, respectively). ${ }^{4,30}$ Finally, Ontario health administrative data does not link records of fathers to their children, and therefore we were uncertain of the fathers' immigration status. However, the overall rate of intermarriage among minority populations in Canada is low (13.0\% in South Asians, $19.4 \%$ in Chinese). ${ }^{31}$

In conclusion, we found that the incidence of IBD among South Asians living in Canada was comparable to the high incidence seen in other Canadians, and significantly higher than incidence rates seen in first-generation immigrants to Canada. Rates in South Asians were much higher than in the Chinese population, even when they were born in Canada. The strong protective effect of immigration to Canada implies that environmental factors to which non-immigrant Canadians of South Asian descent are exposed increase the risk of this immune mediated inflammatory disease. Further research is warranted to understand the pathophysiological reasons for these epidemiological findings, and to understand the health-care needs of the growing numbers of South Asian people with IBD.

\section{Data Sharing Statement}

The data underlying this article was accessed from ICES, which is funded by the Ontario Ministry of Health (MOH) and the Ministry of Long-Term Care (MLTC). The analysis undertaken in this study is based on data and information compiled and provided by: $\mathrm{MOH}$, and the Canadian Institute for Health Information (CIHI). Data sharing is 
governed under a data sharing agreement between ICES and the Ministry of Health and Long-term Care. The derived data generated in this research will be shared on reasonable request to the corresponding author following approval by ICES.

\section{Acknowledgments}

This study was supported by ICES, which is funded by an annual grant from the Ontario Ministry of Health (MOH) and the Ministry of Long-Term Care (MLTC). This study also received funding from the SickKids Inflammatory Bowel Disease Centre. Parts of this material are based on data and information compiled and provided by: $\mathrm{MOH}$, and the Canadian Institute for Health Information (CIHI). The analysis, conclusions, opinions and statements expressed herein are solely those of the authors and do not reflect those of the funding or data sources; no endorsement is intended or should be inferred. Parts or whole of this material are based on data and/or information compiled and provided by Immigration, Refugees and Citizenship Canada (IRCC) current to March 31, 2019. However, the analyses, conclusions, opinions and statements expressed in the material are those of the author (s), and not necessarily those of IRCC.

\section{Author Contributions}

All authors made substantial contributions to conception and design, acquisition of data, or analysis and interpretation of data; took part in drafting the article or revising it critically for important intellectual content; agreed to submit to the current journal; gave final approval of the version to be published; and agree to be accountable for all aspects of the work.

\section{Funding}

Anne Griffiths was supported by the Northbridge Chair in Inflammatory Bowel Diseases.

\section{Disclosure}

No conflicts to declare: Jasbir Dhaliwal, Emily Herrett, Meltem Tuna, Sanjay Murthy, Baiju Shah.

AMG: Anne Griffiths - Consultant: AbbVie, Merck, Janssen, Eli Lilly, Pfizer, Gilead, Roche, Takeda; Speaker: AbbVie, Janssen, Shire; Investigator-initiated research support: AbbVie.

EIB: Eric Benchimol has acted as a legal consultant for Hoffman La-Roche Limited and Peabody \& Arnold LLP for matters unrelated to a medication used to treat inflammatory bowel disease. Dr. Benchimol also acted as a consultant for McKesson Canada. The authors report no other conflicts of interest in this work. The Data Accessed Complies with Relevant Data Protection and Privacy Regulations.

\section{References}

1. Actis GC, Pellicano R, Fagoonee S, et al. History of inflammatory bowel diseases. J Clin Med. 2019;8:1970.

2. Ng SC, Shi HY, Hamidi N, et al. Worldwide incidence and prevalence of inflammatory bowel disease in the 21st century: a systematic review of population-based studies. Lancet. 2018;390:2769-2778. doi:10.1016/S0140-6736(17)32448-0

3. Coward S, Clement F, Benchimol EI, et al. Past and future burden of inflammatory bowel diseases based on modeling of population-based data. Gastroenterology. 2019;156:1345-1353.e4. doi:10.1053/j.gastro.2019. 01.002

4. 2016 census highlights ethnic origin and visible minorities, factsheet 9; 2016.

5. 2016 census highlights immigration, factsheet $8 ; 2016$.

6. Benchimol EI, Mack DR, Guttmann A, et al. Inflammatory bowel disease in immigrants to Canada and their children: a population-based cohort study. Am $J$ Gastroenterol. 2015;110:553-563. doi:10.1038/ajg.2015.52

7. Shah BR, Chiu M, Amin S, et al. Surname lists to identify South Asian and Chinese ethnicity from secondary data in Ontario, Canada: a validation study. BMC Med Res Methodol. 2010;10(1):42. doi:10.1186/1471-2288-10-42

8. Chiu M, Lebenbaum M, Lam K, et al. Describing the linkages of the immigration, refugees and citizenship Canada permanent resident data and vital statistics death registry to Ontario's administrative health database. BMC Med Inform Decis Mak. 2016;16(1):135. doi:10.1186/s12911-016-0375-3

9. The World Bank Country and Lending Groups; 2020.

10. Fitzpatrick T, Wilton AS, Guttmann A. Development and validation of a simple algorithm to estimate common gestational age categories using standard administrative birth record data in Ontario, Canada. J Obstet Gynaecol. 2021;41(2):207-211.

11. Committee PPSS. Provincial Perinatal Report; 2006.

12. Benchimol EI, Guttmann A, Griffiths AM, et al. Increasing incidence of paediatric inflammatory bowel disease in Ontario, Canada: evidence from health administrative data. Gut. 2009;58:1490-1497.

13. Benchimol EI, Guttmann A, Mack DR, et al. Validation of international algorithms to identify adults with inflammatory bowel disease in health administrative data from Ontario, Canada. J Clin Epidemiol. 2014;67:887-896. doi:10.1016/j.jclinepi.2014.02.019

14. Benchimol EI, To T, Griffiths AM, et al. Outcomes of pediatric inflammatory bowel disease: socioeconomic status disparity in a universal-access healthcare system. J Pediatr. 2011;158(6):960-964. doi:10.1016/j.jpeds.2010.11.039

15. Benchimol EI, Kaplan GG, Otley AR, et al. Rural and urban residence during early life is associated with risk of inflammatory bowel disease: a population-based inception and birth cohort study. Am $J$ Gastroenterol. 2017;112(9):1412-1422. doi:10.1038/ajg.2017.208

16. Kralj. Measuring Rurality-RIO2008 Basic: Methodology and Results: Ontario Medical Association; 2008.

17. 2016 Canadian census; 2016.

18. Kaplan GG. The global burden of IBD: from 2015 to 2025. Nat Rev Gastroenterol Hepatol. 2015;12(12):720-727. doi:10.1038/ nrgastro.2015.150

19. Probert CS, Jayanthi V, Pinder D, et al. Epidemiological study of ulcerative proctocolitis in Indian migrants and the indigenous population of Leicestershire. Gut. 1992;33(5):687-693. doi:10.1136/gut.33.5.687 
20. Carr I, Mayberry JF. The effects of migration on ulcerative colitis: a three-year prospective study among Europeans and firstand second- generation South Asians in Leicester (1991-1994). Am J Gastroenterol. 1999;94:2918-2922. doi:10.1111/j.1572-0241.1999. 01438.x

21. Malhotra R, Turner K, Sonnenberg A, et al. High prevalence of inflammatory bowel disease in United States residents of Indian ancestry. Clin Gastroenterol Hepatol. 2015;13(4):683-689. doi:10.1016/j.cgh.2014.06.035

22. Ng SC, Kaplan GG, Tang W, et al. Population density and risk of inflammatory bowel disease: a prospective population-based study in 13 countries or regions in Asia-Pacific. Am J Gastroenterol. 2019;114 (1):107-115. doi:10.1038/s41395-018-0233-2

23. Cleynen I, Boucher G, Jostins L, et al. Inherited determinants of Crohn's disease and ulcerative colitis phenotypes: a genetic association study. Lancet. 2016;387:156-167. doi:10.1016/S0140-6736(15)00465-1

24. Ng SC, Tsoi KKF, Kamm MA, et al. Genetics of inflammatory bowel disease in Asia: systematic review and meta-analysis. Inflamm Bowel Dis. 2012;18(6):1164-1176. doi:10.1002/ibd.21845

25. Leong RW, Armuzzi A, Ahmad T, et al. NOD2/CARD15 gene polymorphisms and crohn's disease in the Chinese population. Aliment Pharmacol Ther. 2003;17:1465-1470. doi:10.1046/j.13652036.2003.01607.x
26. Dhaliwal J, Carroll MW, deBruyn JC, et al. The phenotypic spectrum of new onset IBD in Canadian children of South Asian ethnicity: a prospective multi-centre comparative study. J Crohns Colitis. 2021. doi:10.1093/ecco-jcc/jjab143

27. Piovani D, Danese S, Peyrin-Biroulet L, et al. Environmental risk factors for inflammatory bowel diseases: an umbrella review of meta-analyses. Gastroenterology. 2019;157(3):647-659.e4. doi:10.1053/j.gastro.2019. 04.016

28. de Souza HS, Fiocchi C. Immunopathogenesis of IBD: current state of the art. Nat Rev Gastroenterol Hepatol. 2016;13:13-27. doi:10.1038/nrgastro.2015.186

29. Benchimol EI, Manuel DG, Mojaverian N, et al. Health services utilization, specialist care, and time to diagnosis with inflammatory bowel disease in immigrants to Ontario, Canada: a Population-Based Cohort Study. Inflamm Bowel Dis. 2016;22:2482-2490. doi:10.1097/ MIB.0000000000000905

30. 2006 census ethnic origin and visible minorities, factsheet $11 ; 2006$.

31. Mixed unions in Canada; 2011. Available from: https://www12.stat can.gc.ca/nhs-enm/2011/as-sa/99-010-x/99-010-x2011003_3-eng. cfm. Accessed November 15, 2021.
Clinical Epidemiology

\section{Publish your work in this journal}

Clinical Epidemiology is an international, peer-reviewed, open access, online journal focusing on disease and drug epidemiology, identification of risk factors and screening procedures to develop optimal preventative initiatives and programs. Specific topics include: diagnosis, prognosis, treatment, screening, prevention, risk factor modification,

Submit your manuscript here: https://www.dovepress.com/clinical-epidemiology-journal

\section{Dovepress}

systematic reviews, risk \& safety of medical interventions, epidemiology \& biostatistical methods, and evaluation of guidelines, translational medicine, health policies \& economic evaluations. The manuscript management system is completely online and includes a very quick and fair peer-review system, which is all easy to use. 